\title{
The Establishment and Operation of Provincial Key Laboratories Open Fund Management System---- A case Study of Jinan University
}

\author{
Wang Hailan ${ }^{1, a}$, Li Yanfei ${ }^{2, b}$, Wang Zhao ${ }^{1, c}$, Tang Minghui ${ }^{1, d}$ \\ ${ }^{1}$ Department of Science and Technology, Jinan University, Guangzhou, 510632, China \\ ${ }^{2}$ Department of International Economics and Trade, Guangdong University of Foreign Studies \\ South China Business College, Guangzhou, 510545, China \\ aemail: 58152287@qq.com, bemail:442897112@qq.com, cemail:360864031@qq.com, \\ demail:909770042@qq.com
}

Keywords: Provincial Key Laboratories; Open Fund; Management Mode

\begin{abstract}
This paper takes Optoelectronic Information and Sensing Technology Key Laboratory in Jinan University for the study, do a research on the key laboratories open fund management and operation mechanism. Then give an analysis of provincial key laboratories' features and their inadequacies. At last give some recommendation on the open fund management and operation.
\end{abstract}

\section{Introduction}

In recent years, Department of Science and Technology, Ministry of Education and universities jointly have established a number of provincial key laboratories. Such key laboratories, mainly engaged in basic and applied research, are useful supplement of national science and technology innovation system.

Up to the early of 2013, Jinan University has established three ministerial key laboratories, 10 provincial key laboratories. However, due to the different areas of laboratory research, there are big differences on the provincial key Laboratory management mode. In order to further strengthen the provincial key laboratory construction, Jinan University began to support greater special funds for the basic research laboratory. To seek more reasonable, optimized, and universal management mode, this paper will take a discussion on the management mode of open fund under the new situation.

2 The Characteristics of Provincial Key Laboratory

(1) Encourage Curiosity-Driven Exploration and Research

Open Fund, which funding is from the provincial and ministerial key laboratories operating funds, is mainly funding for research in the field of scientific issues, encouraging cross and exploration. Although in recent years the local government has increased funding for key laboratory, but still has a limit on the funding. Because laboratory maintenance includes laboratory operating funds in fuel and power, operation and maintenance of public facilities, laboratories academic conferences, hiring temporary staff, etc. But the general research funding for each project is about 20 to 40 thousand. For this feature, open fund seeks to achieve concise, mainly used to encourage curiosity-driven exploration and research.

\section{(2) Strictly Selecting Topics and Researchers}

There are four ways for the open fund of provincial key laboratory in Jinan University. First is provided by the laboratory of funding, applicants done alone; second is provided by the laboratory of funding, applicants use room equipment and other research conditions; third is to use own funds or to establish the sharing funds, use the laboratory equipment and other key conditions for scientific research; fourth is to participate in laboratory studies which has been included in the key scientific research projects. Since the first two types are easy management, as well as the results marked secret is relatively simple and therefore a wider application. Selecting appropriate topics and researchers is to approve one of open issues, not only can ensure the open funds can get good results, but also add further laboratory research, and promote academic development.

3 The Inadequacies of Provincial Key Laboratory Project Management

(1) Narrow Opening Fund Applications

Each laboratory has many research directions and contents. As the management of open fund 
propaganda is not enough, the use of propaganda is not strong, resulting in the application of fund projects tend to be limited in a certain direction and content, without taking into account the directions and contents of the various researches. What we should do is to help applicants to broaden application field and research.

\section{(2) The Assessment Process of Open Fund not Rigorous Enough}

Applicants are very serious for applying for funds. As fund managers, regardless of how the applicant's level, we all want fund's assessment should strictly abide by the "just, fair and open" principle. Open fund assessment process compliances with the basic principles, but because of objective reasons, there have been a number of disturbing factors affecting the assessment results to a certain extent. For the assessment results, the losers did not get adequate reasons for losing and aren't aware of their shortcomings, this does not help them to prepare for the next application.

\section{(3) Open Fund Management Not Perfect in the Late}

In general, the applicants and managers of the fund are seriously about the period of pre-application and approval, but once the application is approved, both pay less attention in the late management. There are many problems in the late management. First, lacking communication with project leaders in a timely manner. Not timely understanding of the projects progress will lead to failure to give timely reminds. Secondly, in the late research, there is not enough time for date collection. This situation will affect the effect.

\section{(4) Lack of Tracking Management on the Open Fund}

Tracking management of the fund, also called real-time management of the fund, should be one of the most effective means on the project management. Because of the lack of management tools, we can't grasp the dynamic information of project leader, even more impossible to have a real-time record of achievements, so it is likely to affect the effect.

\section{A Case Study in Jinan University}

Optoelectronic Information and Sensing Technology Laboratory in Jinan University, which was approved the establishment in December 2008 by the Guangdong Provincial Department of Education, has passed the acceptance in December 2012. It is in accordance with the "open, mobile, union, competition" mechanism. In the short period of four years, it has formed its own characteristics and suitable development management mode.

\subsection{Expanding Sphere of Influence, Try for more Applications}

(1) Increase the open fund propaganda. There are three ways to propagate. Network propaganda, it means to list the special column and publish application guide on the website of the key laboratory. Human propaganda, members of the laboratory do the publicity when they attend national and international conferences. Cordially invitation to researchers abroad, in order to expand the research room and promote the development of cross-disciplinary, laboratories invite them to work in the team. At the same time, to strengthen communication and cooperation with other universities' laboratories in order to form powerful alliances and get better quality results.

(2) Increase funding for research projects. The key laboratory publishes the application guide information on the website. It funds 100 thousand in support of research projects. From 2009, the number of fund applications is constantly increasing and it has achieved good results.

\subsection{Take the Avoidance and Confidentiality Measures for Assessment}

To ensure fair and just, the internal members of the key laboratory could not apply for the open fund, the applicants should be the non-laboratory researchers or post-doctor. Meanwhile, in order to avoid the difficulty of project establishment, it requires applicants must join a laboratory researcher jointly apply.

Selecting the appropriate topic and suitable applicants are important guarantee factor, therefore, open projects need to go through three procedures to determine whether it can support. The first is the formal examination. Those fail to pass the examinations couldn't get the funding. For the relatively innovative research topic, we will contact with the applicants to do the suitable modify. And then the chief researcher of the laboratory will assess the application, score, and submit to the Academic Committee proportionally. Finally, the Academic Committee will discuss and vote for the result. 


\subsection{Standardized Achievement Management, Clear Intellectual Property}

Generally, the time for open project is two years. It will take some time from research to summarize form words, and publication. The lab adopts the tracking management. It means that we will evaluate the project achievements after one year when finishing the project.

Another measure is to standardize the results tagging, fund project related papers, monographs, achievements appraisal information, etc., should be marked "Optoelectronic Information and Sensing Technologies of Guangdong Provincial Key Laboratory of Research Fund project" and the project number.

Interim inspection is in the form of academic communication. Project leaders need to conduct an interim report on the project. The reporting form is not taking simple questions and answers, but requires the project leader to do the academic communicate with experts and scholars face to face. This will help to explore more questions and new ideas.

Fund post-administration is a weak link. In the future, development can be based on computer and network platform technology to improve the management of project, public participation, results-based management of the normative and project management performance. Meanwhile, to pay more focus on training researcher's management level, improving their management skills and innovative consciousness.

\subsection{Strengthen the Fund Management to Create Distinctive Culture}

Fund management is science, but also culture. Focus on laboratory culture, creating a strong academic atmosphere, using its own charm to attract researchers to carry out the laboratory work is also an important element for the efficient use of special funds laboratory and a high level of research results.

Optoelectronic Information and Sensing Technology Laboratory organize academic meetings once every two months. It provides good opportunity for researchers to share their new ideas and thinking. This not only stimulates creativity, but also enhances the cohesion of the research team, in research to achieve cross, integration and innovation.

In four years, Optoelectronic Information and Sensing Technologies Key Laboratory set up nine projects, the total project budget of one hundred eighty thousand RMB, of which six projects have been concluding acceptance. Open fund project has made great achievements in the SCI journals published 21 papers, 12 of EI and core journals, 8 patents, really a high level of achievement results.

\section{Conclusion}

The management of open fund should focus more on people-oriented management philosophy. Only by strengthening management and communication between the project managers and leaders in order to fully and effectively mobilize the enthusiasm of the project leader. Attract more talented people to do the basic research through the open fund .This is better for the development of science, technology and economic. Project supported by Fifteenth Installment of Teaching Reform Project, Jinan University(No.JG2013044)

\section{References}

[1] Dong Cheng, Zhang Yu Ying, Zuo HaoWang. U.S. Federal Laboratory Performance Evaluation and its Reform[J]. Experimental Technology and Management, 2006, 23 (11): 1-6.

[2] Huang Chunji, Liu Weidong. Strengthen the National Natural Science Foundation Process Management Practice and Thinking [J]. Journal of Medical Science Research Management, 2005, 18 (4): 211 - 212.

[3] Ma Tong, etc. to promote open sharing of large equipment to improve resource efficiency equipment [J]. Experimental Technology and Management, 2005, 22 (6) :128-131.

[4] Su Rongjun, Liu Ning, Li Jian, etc.. Raise Chemistry Experimental Teaching Practice and Exploration [J]. Laboratory Science, 2009 (5): 182 - 184.

[5] Zhang Rongde, Qin Falan. Research on Characteristics of State Key Laboratory[J]. Laboratory Research and Exploration, 2001, 20 (5): 96-97. 